\title{
Magnetic droplet solitons in orthogonal spin valves
}

\author{
Sunjae Chung ${ }^{1,2}$, S. Majid Mohseni ${ }^{3}$, Anders Eklund ${ }^{4}$, Philipp Dürrenfeld ${ }^{1}$, Mojtaba Ranjbar ${ }^{1}$, \\ Sohrab R. Sani ${ }^{2}$, T. N. Anh Nguyen ${ }^{2,5}$, Randy K. Dumas ${ }^{1}$, and Johan Åkerman ${ }^{1,2}$ \\ ${ }^{1}$ Department of Physics, University of Gothenburg, 41296 Gothenburg, Sweden \\ ${ }^{2}$ Department of Materials and Nanophysics, School of Information and Communication Technology, KTH Royal In- \\ stitute of Technology, Electrum 229, 16440 Kista, Sweden \\ ${ }^{3}$ Department of Physics, Shahid Beheshti University, Tehran 19839, Iran \\ ${ }^{4}$ Department of Integrated Devices and Circuits, School of Information and Communication Technology, \\ KTH Royal Institute of Technology, Electrum 229, 16440 Kista, Sweden \\ ${ }^{5}$ Lab of Magnetism and Superconductivity, Institute of Materials Science, Vietnam Academy of Science and Tech- \\ nology, 18 Hoang Quoc Viet, Cau Giay, Hanoi, Vietnam \\ E-mail: johan.akerman@physics.gu.se
}

Received July 30, 2015, published online August 25, 2015

\begin{abstract}
We review the recent experimental advancements in the realization and understanding of magnetic droplet solitons generated by spin transfer torque in orthogonal nanocontact based spin torque nanooscillators (STNOs) fabricated on extended spin valves and spin valve nanowires. The magnetic droplets are detected and studied using the STNO microwave signal and its resistance, the latter both quasistatically and time-resolved. The droplet nucleation current is found to have a minimum at intermediate magnetic field strengths and the nature of the nucleation changes gradually from a single sharp step well above this field, mode-hopping around the minimum, and continuous at low fields. The mode-hopping and continuous transitions are ascribed to droplet drift instability and re-nucleation at different time scales, which is corroborated by time-resolved measurements. We argue that the use of tilted anisotropy fixed layers could reduce the nucleation current further, move the nucleation current minimum to lower fields, and potentially remove the need for an applied magnetic field altogether. Finally, evidence of an edge mode droplet in a nanowire is presented.

PACS: 75.30.Ds Spin waves;

75.75.-c Magnetic properties of nanostructures.
\end{abstract}

Keywords: magnetic droplet soliton, spin torque, perpendicular anisotropy, magnetoresistance.

\section{Introduction}

The phenomenon of spin transfer torque (STT) [1-3], in which angular momentum can be transferred from a spin polarized current to a magnetic layer, has dramatically impacted how magnetodynamics can be excited in magnetic nanostructures. As STT can act as negative spin wave damping, it can realize magnetic systems where spin waves, instead of being damped out, can grow exponentially in amplitude, until additional non-linear damping balances the magnetodynamics and a steady state of intense spin wave generation is realized [4-6]. This auto-oscillatory state is the basis for spin torque nanooscillators (STNOs) with promise for use as ultra-broadband, rapidly modulated, truly nanoscopic, and RF CMOS compatible microwave signal generators [5-17].

While STNOs can be successfully fabricated in the form of $100 \mathrm{~nm}$ diameter nanopillars of either spin valve [18] or magnetic tunnel junction [19] stacks, nanocontact based STNOs [20,21], in which the active magnetic layers are laterally extended over several micrometers and only the nanocontact is nanoscopic, typically offer much richer magnetodynamics, entirely novel magnetodynamic modes, and even magnetic solitons [22-25]. For example, in easyplane material based devices such as STNOs with Permalloy free layers, in addition to vortices [26-28], both propa- 
gating spin waves [29-36] and self-localized spin wave bullet solitons [31-33,37] can be generated experimentally.

Very recently, orthogonal spin valve STNOs with free layers having perpendicular magnetic anisotropy were realized $[38,39]$. While originally developed to achieve zerofield operation, these STNOs were later shown to be able to nucleate and sustain so-called magnetic droplet solitons [40-44], which are the dissipative analog of the magnon drop solitons suggested in the 1970s [22,24]. While the magnetic droplet is of great fundamental interest in itself, it also promises significant advantages in both microwave and memory applications. As the magnetic droplet typically exhibits a partially reversed core a large fraction of its spins precess at a very large angle and make use of much more of the available magnetoresistance than many other modes. While typical STNO precession angles reach a maximum of about 20 degrees [45], and hence generate only about $10 \%$ of the theoretical maximum microwave power, droplets can ideally be made to precess close to 90 degrees and consequently generate close to the maximum available power. In memory applications, it has also been shown that droplets are the necessary precursor to skyrmion injection into skyrmion based race track memories [46].

In this paper we review the recent progress in realizing magnetic droplets in different geometries and provide a better understanding of some of their fundamental properties such as their nucleation and collapse boundaries. We show magnetic droplet nucleation versus applied magnetic field and electrical drive current. Furthermore, we argue that the use of tilted anisotropy materials for the fixed layer could greatly reduce the current and magnetic field required for magnetic droplet nucleation. Finally, the time dependent resistance measurements of droplet collapse and re-nucleation at high field strengths will be presented and discussed as well as droplet nucleation in nanowires, as required for future racetrack memories.

\section{Experimental}

The orthogonal spin valve stacks, Fig. 1(a), based on $\mathrm{Ta}(4 \mathrm{~nm}) / \mathrm{Cu}(10 \mathrm{~nm}) / \mathrm{Ta}(4 \mathrm{~nm}) / \mathrm{Co}(6 \mathrm{~nm}) / \mathrm{Cu}(6 \mathrm{~nm}) /$ $\mathrm{Co}(0.2 \mathrm{~nm})-[\mathrm{Ni}(0.6 \mathrm{~nm}) / \mathrm{Co}(0.25 \mathrm{~nm})] \times 4$ were magnetron sputtered at room temperature on thermally oxidized Si substrates in a chamber with a base pressure better than $5 \cdot 10^{-8}$ Torr. The sputtering growth rates used for the ultrathin Co and Ni layers were less than $0.2-0.3 \AA / s$ to maximize uniformity and minimize inter-diffusion. Using alternating gradient magnetometry we estimated the in-plane saturation field $\mu_{0}\left(H_{k}-M_{S}\right)$ of the free layer to be $0.35 \mathrm{~T}$.

The subsequent fabrication of the STNOs includes the following steps: (i) patterning the blanket films to either a $8 \times 16 \mu \mathrm{m}$ mesa using optical lithography or $200 \mathrm{~nm}$ wide nanowires using $e$-beam lithography, (ii) deposition of an insulating $\mathrm{SiO}_{2}$ layer by chemical vapor deposition, (iii) defining the NC area using electron-beam lithography, (iv) re-

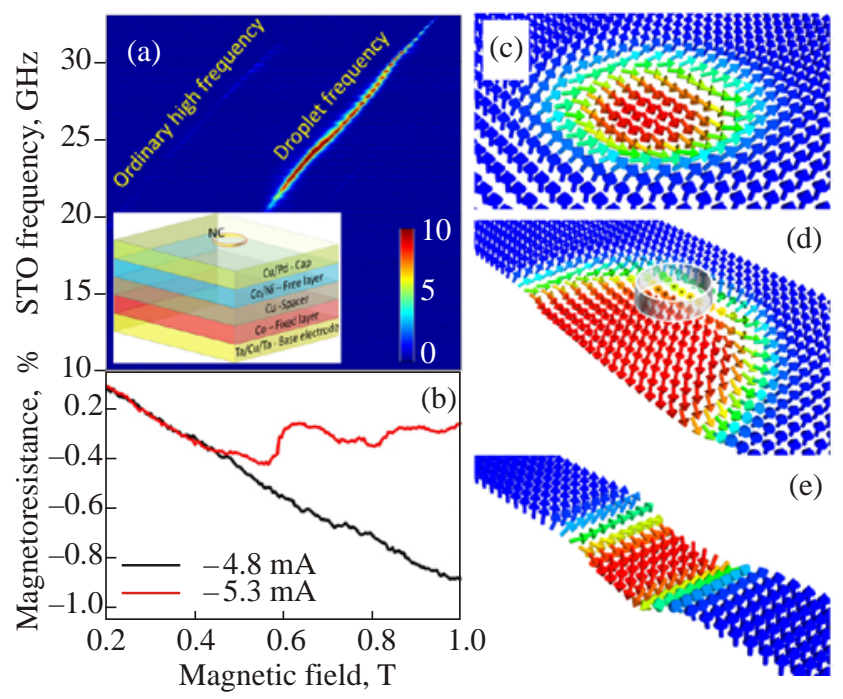

Fig. 1. (Color online) The inset in (a) shows a schematic geometry of the orthogonal spin valve stack and a nanocontact (NC). A transition from an ordinary high frequency mode to a magnetic droplet is evident as a dramatic drop in frequency (a) and increase in magnetoresistance (b), (red curve) as the applied field is increased. As a reference in (b), (black curve) the magnetoresistance only shows a monotonic decrease for currents less than the necessary nucleation current. (c)-(e) Snapshots from micromagnetic simulations showing the spin configurations of a (c) conventional magnetic droplet nucleated in an extended free layer, (d) an edge-mode magnetic droplet nucleated in a nanowire, and (e) a quasi-1D droplet consisting of two precessing domain walls which exhibit a well-defined breathing.

active ion etching through the $\mathrm{SiO}_{2}$ to define the NC, (v) deposition of a top contact electrode of $\mathrm{Cu}(1.1 \mu \mathrm{m}) / \mathrm{Au}$ $(100 \mathrm{~nm})$, and (vi) forming the electrodes into a co-planar waveguide using optical lithography and lift-off.

A custom probe station was used for magnetoelectrical characterization of the STNOs utilizing applied fields perpendicular to the film stack. The generated microwave signal was amplified using a broadband microwave amplifier and measured in the frequency domain with a spectrum analyzer. The dc voltage was simultaneously measured across the device for magnetoresistance (MR) measurements.

Time dependent STNO resistance measurements were carried out via the $0-30 \mathrm{kHz}$ dc port of the bias-T using a EG\&G 5113 low-noise amplifier utilizing ac coupling, $30 \mathrm{~dB}$ voltage gain and a $0-10 \mathrm{kHz}$ bandwidth.

\section{Results and discussions}

\subsection{Magnetic droplet nucleation current vs field}

We will first discuss the results related to magnetic droplets in extended films. Figures 1(a) and 1(b) show typical microwave and magnetoresistance measurements of magnetic droplet nucleation as a function of applied field 
at a fixed current. These extended layers support the traditional magnetic droplet soliton, depicted schematically in Fig. 1(c), where the core is partially reversed and the spins both in the core and in the droplet perimeter precess inphase. Although droplet theory [40] was developed for fully perpendicular spin valves, reported experiments $[41,44]$ have used STNOs where the remanent state of the fixed layer is in the film plane, which can promote droplet instability and auto-modulation [41,47-49]. In this orthogonal geometry, the current-field phase diagram of the droplet qualitatively differs from the theoretical predictions. Although experiments and theory agree at high fields where the fixed layer is saturated out-of-plane [44], the low-field behavior of the droplet nucleation current instead scales well with the perpendicular component of the spin polarization [41]. At intermediate fields both these descriptions appear to break down [42].

To investigate these different behaviors in detail, we study droplet nucleation over a wider field range in a STNO with a $90 \mathrm{~nm}$ diameter NC. Figure 2 shows the STNO resistance vs current for fields ranging from 1.1 to $1.8 \mathrm{~T}$, where the formation of the droplet can be monitored as a step-like increase in the resistance, as indicated with triangle symbols. There is a clear minimum in the nucleation current at intermediate fields, which connects the two different dependencies observed in Refs. 41 and 44. We as-

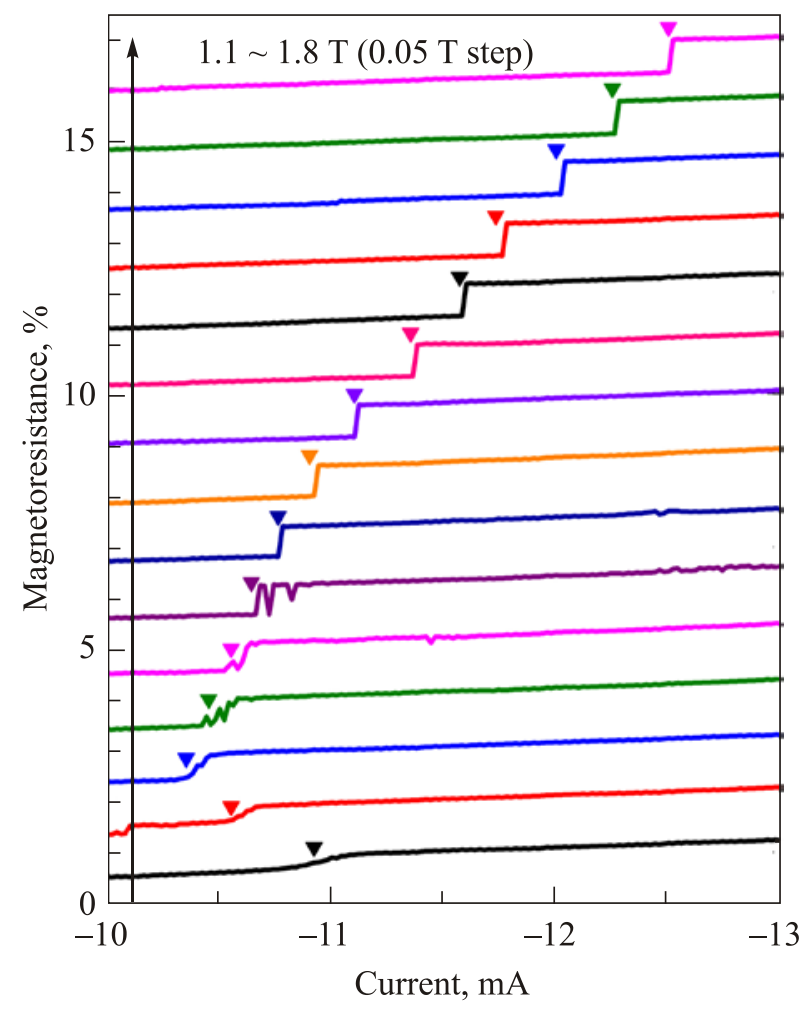

Fig. 2. (Color online) Resistance vs applied current at different external out-of-plane fields between 1.1 and 1.8 T. Plots are vertically shifted for clarity. The droplet nucleation current is depicted with a triangle in each measurement. cribe the high field linear behavior to the usual linear field dependence of reaching the Slonczewski spin wave instability in spin torque devices [29,50]. The low-field regime, where the nucleation current has been found to be inversely proportional to the applied field [41, 42] is instead a consequence of the gradual out-of-plane tilting of the fixed layer. The primary limiting factor to reach the Slonczewski spin wave instability in this regime is the amount of perpendicular spin polarization in the STNO current.

Finding the minimum nucleation current is important for applications as one typically would like to reduce the power consumption. The decreasing perpendicular spin polarization at low fields is therefore undesirable. It would be advantageous if one could use both a low field and a low current and still be able to nucleate a magnetic droplet. While a fully perpendicular STNO, such as that assumed in the original prediction of droplets [40], would have the lowest nucleation current, it would on the other hand also not have any output signal, since the symmetry of the magnetodynamics would not generate a time-varying STNO resistance. Instead we argue that a fixed layer based on tilted anisotropy materials [51-60] would be ideal for droplet nucleation and operation. In Fig. 3 we schematically compare the present STNOs with a STNO based on a tilted anisotropy fixed layer. Since the magnetization angle at the top of the tilted exchange spring can be tuned by tailoring the individual thicknesses of the perpendicular and easy-plane layers respectively, it will be possible to optimize this system for both zero-field operation and droplet nucleation at a minimal current. We consequently expect significant a research effort in this direction to be carried out soon.

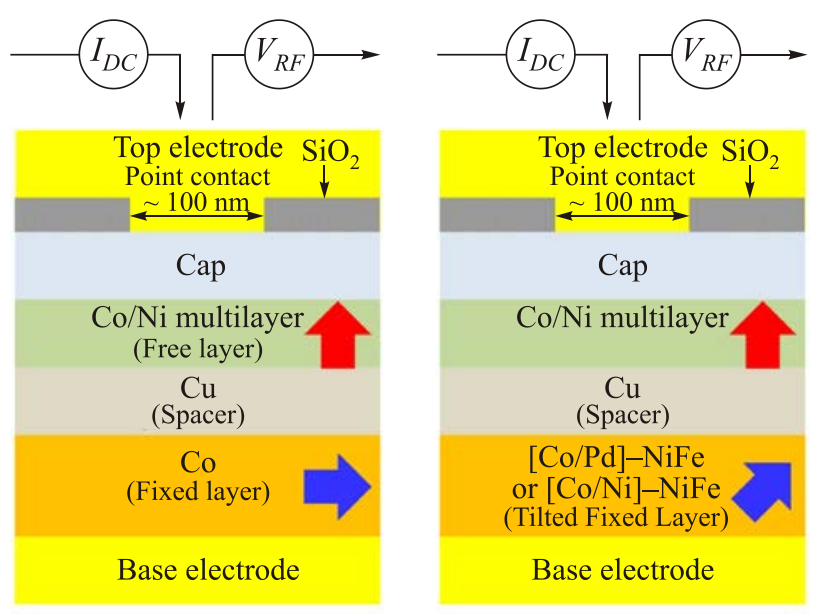

Fig. 3. (Color online) The left schematic shows the original orthogonal stack in which magnetic droplets were first discovered. In the right schematic, the fixed layer has been replaced with a tilted exchange spring, e.g. [Co/Pd]-NiFe, in which the remanent out-of-plane angle of the NiFe spins can be freely tailored. As described in the text, this is expected to remove any need for an applied field to observe droplets. 


\subsection{Magnetic droplet collapse and re-nucleation}

It can be seen in Fig. 2 that the droplet nucleation as a function of current is step-wise at high fields, more gradual at the lowest fields, and shows a mode-hopping behavior at intermediate fields. The mechanism responsible for this phenomenon is most likely the drift instability predicted in the original numerical droplet demonstration [40]. At the high field end of Fig. 2 the droplet appears entirely stable and a single well-defined nucleation event triggers the resistance step. At intermediate fields, where the tilt angle of the fixed layer is smaller, the drift instability becomes active but is still relatively rare, such that a small number of drift instability events occur during the initial current ramp. However, as the current is well above the nucleation threshold, a new droplet immediately re-nucleates and this appears like discrete mode-hopping. Finally, at the lowest fields, there is neither a sharp step nor any signs of modehopping. Instead there is a smooth gradual resistance change. We interpret this as the mean time between drift instability events being much shorter than the integration time of the resistance measurement. As the STNO is again above the nucleation threshold, every drift instability event is immediately followed by a droplet re-nucleation and the resistance measurement now reflects the ratio between the STNO being in a droplet state or in a uniform state. This ratio manifests itself as a smooth function of current.

We have previously shown that droplet collapse at high fields can exhibit similar signs of mode hopping [43]. In Fig. 4 we present time-dependent resistance measurements of a different STNO at high perpendicular field using a real-time sampling oscilloscope to record long timetraces at high temporal resolution. At the operating current of $-6.4 \mathrm{~mA}$ the collapse field as measured by ordinary resistance and microwave signal generation is about $1.77 \mathrm{~T}$. However, when the same device is measured using the oscilloscope, one can clearly observe sharp transitions between a high and a low resistance state. At $1.75 \mathrm{~T}$, the dominating state is the droplet, whereas at $1.79 \mathrm{~T}$, the uniform state has almost entirely replaced it. At $1.77 \mathrm{~T}$ the times spent in the droplet and uniform state, respectively, are more equal.

\subsection{Magnetic droplets in nanowires}

We finally turn to droplets in reduced dimensions. According to previous micromagnetic simulations [61] the droplet in an extended free layer, Fig. 1(c), undergoes a transition to an edge droplet mode, Fig. 1(d), when the nanowire has a width between 2-3.5 times the NC diameter. This edge droplet mode is a consequence of the attraction from the nanowire boundary [62]. Additionally, the edge droplet has a much larger mode volume, which is governed by the nanowire width rather than the NC size, yielding an effectively lower oscillation frequency [40]. For nanowire widths smaller than twice the NC diameter, the micromagnetic simulations showed the existence of
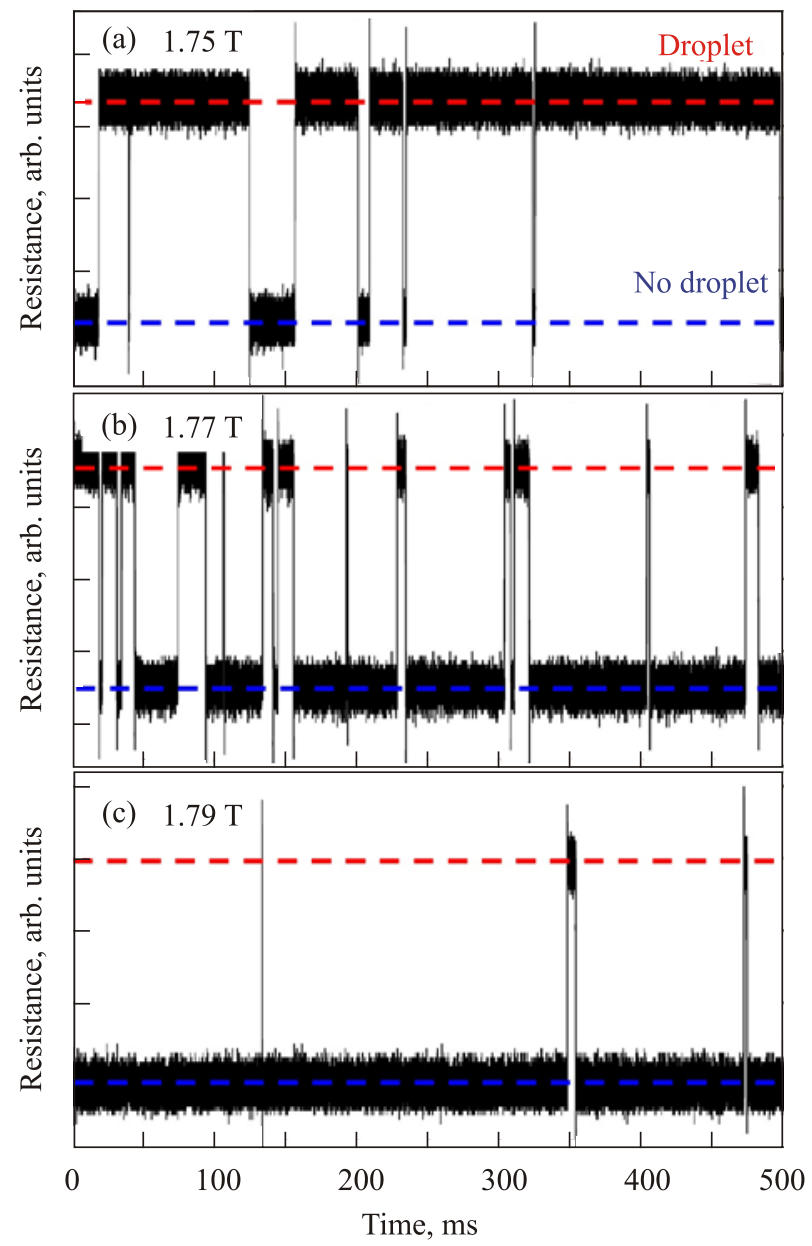

Fig. 4. (Color online) Time dependent STNO resistance measurements at $I=-6.4 \mathrm{~mA}$ in three slightly different perpendicular fields, $\mathrm{T}$ of $1.75 \mathrm{~T}$ (a), 1.77 (b) and 1.79 (c). The highresistance state indicates the presence of a magnetic droplet, whereas the low-resistance state indicates a uniform, non-droplet, magnetic state. The over- and undershooting spikes stem from the amplifier low-pass filter.

a quasi one-dimensional droplet mode, Fig. 1(e), where the expansion of the dynamics across the nanowire width results in a breathing pair of domain walls with subZeeman oscillation frequencies.

A scanning electron micrograph of a $200 \mathrm{~nm}$ wide nanowire spin valve structure with a NC diameter of $70 \mathrm{~nm}$ is shown in the upper inset of Fig. 5. The STNO frequency spectra as a function of the applied perpendicular field is shown in the main panel of Fig. 5. The frequency is below the FMR frequency for all conditions, consistent with the formation of a droplet. However, at a field of $0.7 \mathrm{~T}$, a sharp decrease of the oscillation frequency by $1 \mathrm{GHz}$ is observed. A similar drop in frequency is also present in a current sweep at the constant field of $0.75 \mathrm{~T}$, as shown in the lower inset of Fig. 5. We believe that this drop in frequency is consistent with the formation of the edge mode, where the droplet extends in an arc from the NC to the nanowire edge. These first experimental results demon- 




Fig. 5. (Color online) Power spectral density vs perpendicular field at a constant current of $-12 \mathrm{~mA}$ for a nanowire based STNO. The top inset shows a SEM image of the $200 \mathrm{~nm}$ wide nanowire including the etched hole for the final NC. The bottom inset shows the power spectral density vs current in a constant perpendicular field of $0.75 \mathrm{~T}$.

strating the nucleation of droplets in nanowires are especially promising for the implementation of confined magnetic solitons into nanostructured devices, e.g. racetrack memories.

\section{Conclusions}

In summary, we have presented a brief review of the most recent experiments and advances related to magnetic droplet solitons in orthogonal spin valves. In particular, we provided a detailed investigation regarding droplet nucleation over a wide range of currents and fields. Not only did we observe a clear minimum in the nucleation current, but were also able to resolve important differences in exactly how a droplet nucleates at different applied fields. For example, at large applied fields droplet nucleation occurs by a single well-defined event. However, for smaller fields this transition begins to blur as the role of the drift instability promotes more complex nucleation/re-nucleation dynamics, which is also evident in additional time-resolved measurements. Our latest measurements on droplet nucleation in orthogonal spin valve nanowires provides the first experimental evidence of fundamentally new droplet dynamics, namely a unique droplet edge-mode, where the physical boundaries of the nanowire begin to play an important role on the overall magnetodynamics. Future prospects include the use of tilted fixed layers, which will allow for further optimization of the dynamics and potentially zero-field operation, and skyrmion nucleation in nanowires for racetrack memories, both of which will be useful for next generation spintronic and magnonic device applications.
1. L. Berger, Phys. Rev. B 54, 9353 (1996).

2. J.C. Slonczewski, J. Magn. Magn. Mater. 159 L1 (1996).

3. D. Ralph and M. Stiles, J. Magn. Magn. Mater. 320, 1190 (2008).

4. A. Slavin and V. Tiberkevich, Magnetics, IEEE Transactions 45, 1875 (2009).

5. J.V. Kim, Solid State Phys. 63, 217 (2012).

6. T. Silva and W. Rippard, J. Magn. Magn. Mater. 320, 1260 (2008).

7. M.R. Pufall, W.H. Rippard, S. Kaka, T.J. Silva, and S.E. Russek, Appl. Phys. Lett. 86, 082506 (2005).

8. A. Eklund, S. Bonetti, S.R. Sani, S. Majid Mohseni, J. Persson, S. Chung, S. Amir Hossein Banuazizi, E. Iacocca, M. Östling, J. Åkerman, and B. Gunnar Malm, Appl. Phys. Lett. 104, 092405 (2014).

9. J. Persson, S.R. Sani, S. Bonetti, F. Magnusson, Y. Pogorylov, S.M. Mohseni, S. Gunnarsson, M. Norling, C. Stoij, and J. Åkerman, IEEE Trans. Magn. 48, 4378 (2012).

10. P.K. Muduli, Y. Pogoryelov, Y. Zhou, F. Mancoff, and J. Åkerman, Integr. Ferroelectr. 125, 147 (2011).

11. P.K. Muduli, Y. Pogoryelov, S. Bonetti, G. Consolo, F. Mancoff, and J. Åkerman, Phys. Rev. B 81, 140408(R) (2010).

12. Y. Pogoryelov, P.K. Muduli, S. Bonetti, E. Iacocca, F. Mancoff, and J. Åkerman, Appl. Phys. Lett. 98, 192501 (2011).

13. Y. Pogoryelov, P.K. Muduli, S. Bonetti, F. Mancoff, and J. Åkerman, Appl. Phys. Lett. 98, 192506 (2011).

14. P.K. Muduli, O.G. Heinonen, and J. Åkerman, Phys. Rev. B 86, 174408 (2012).

15. P.K. Muduli, Y. Pogoryelov, F. Mancoff, and J. Åkerman, IEEE Trans. Magn. 47, 1575 (2011).

16. Y. Pogoryelov, P.K. Muduli, and J. Åkerman, IEEE Trans. Magn. 48, 4077 (2012).

17. T. Chen, A. Eklund, S. Sani, S. Rodrigueza, G. Malm, J. Åkerman, and A. Rusu, Solid State Electronics 111, 91 (2015).

18. V.S. Pribiag, I.N. Krivorotov, G.D. Fuchs, P.M. Braganca, O. Ozatay, J.C. Sankey, D.C. Ralph, and R.A. Buhrman, Nat. Phys. 3, 498 (2007).

19. I.N. Krivorotov, N.C. Emley, J.C. Sankey, S.I. Kiselev, D.C. Ralph, and R.A. Buhrman, Science 307, 228 (2005).

20. W. Rippard, M. Pufall, S. Kaka, S. Russek, and T. Silva, Phys. Rev. Lett. 92, 027201 (2004).

21. R. Dumas, S. Sani, S. Mohseni, E. Iacocca, Y. Pogoryelov, P. Muduli, S. Chung, P. Dürrenfeld, and J. Åkerman, Magnet., IEEE Transact. 50, 1 (2014).

22. B. Ivanov and A. Kosevich, Zh. Eksp. Teor. Fiz. 72, 2000 (1977).

23. A. Kovalev, A. Kosevich, and K. Maslov, JETP Lett. 30, 296 (1979).

24. A. Kosevich, B. Ivanov, and A. Kovalev, Phys. Rep. 194, 117 (1990).

25. H.-B. Braun, Adv. Phys. 61, 1 (2012).

26. T. Devolder, A. Meftah, K. Ito, J.A. Katine. P. Crozat, and C. Chappert, J. Appl. Phys. 101, 063916 (2007). 
27. S.R. Sani, J. Persson, S.M. Mohseni, V. Fallahi, and J. Åkerman, J. Appl. Phys. 109, 07C913 (2011).

28. S.R. Sani, P. Durrenfeld, S.M. Mohseni, S. Chung, and J. Åkerman, IEEE Trans. Magn. 49, 4331 (2013).

29. M. Tsoi, A. Jansen, J. Bass, W. Chiang, V. Tsoi, and P. Wyder, Nature 406, 46 (2000).

30. M. Madami, S. Bonetti, G. Consolo, S. Tacchi, G. Carlotti, G. Gubbiotti, F.B. Mancoff, M.A. Yar, and J. Åkerman, Nature Nanotech. 6, 635 (2011).

31. S. Bonetti, V. Tiberkevich, G. Consolo, G. Finocchio, P. Muduli, F. Mancoff, A. Slavin, and J. Åkerman, Phys. Rev. Lett. 105, 217204 (2010).

32. S. Bonetti, V. Puliafito, G. Consolo, V.S. Tiberkevich, A.N. Slavin, and J. Åkerman, Phys. Rev. B 85, 174427 (2012).

33. R.K. Dumas, E. Iacocca, S. Bonetti, S.R. Sani, S.M. Mohseni, A. Eklund, J. Persson, O. Heinonen, and J. Åkerman, Phys. Rev. Lett. 110, 257202 (2013).

34. F.B. Mancoff, N.D. Rizzo, B.N. Engel, and S. Tehrani, Nature 437, 393 (2005).

35. S. Kaka, M.R. Pufall, W.H. Rippard, T.J. Silva, S.E. Russek, and J.A. Katine, Nature 437, 389 (2005).

36. S.R. Sani, J. Persson, S.M. Mohseni, Y. Pogoryelov, P.K. Muduli, A. Eklund, G. Malm, M. Käll, A. Dmitriev, and J. Åkerman, Nat. Commun. 4, 2731 (2013).

37. V.E. Demidov, S. Urazhdin, and S.O. Demokritov, Nature Mater. 9, 984 (2010).

38. W.H. Rippard, A.M. Deac, M.R. Pufall, J.M. Shaw, M.W. Keller, S. E. Russek, and C. Serpico, Phys. Rev. B 81, 014426 (2010).

39. S.M. Mohseni, S.R. Sani, J. Persson, T.N. Anh Nguyen, S. Chung, Y. Pogoryelov, and J. Åkerman, Phys. Status Solidi Rapid Res. Lett. 5, 432 (2011).

40. M.A. Hoefer, T.J. Silva, and M.W. Keller, Phys. Rev. B 82, 054432 (2010).

41. S.M. Mohseni, S.R. Sani, J. Persson, T.N. Anh Nguyen, S. Chung, Y. Pogoryelov, P.K. Muduli, E. Iacocca, A. Eklund, R.K. Dumas, S. Bonetti, A. Deac, M.A. Hoefer, and J. Åkerman, Science 339, 1295 (2013).

42. S.M. Mohseni, S.R. Sani, R. Dumas, J. Persson, T.N. Anh Nguyen, S. Chung, Y. Pogoryelov, P. Muduli, E. Iacocca, A. Eklund, and J. Åkerman, Physica B 435, 84 (2014).

43. S. Chung, S.M. Mohseni, S.R. Sani, E. Iacocca, R.K. Dumas, T.N. Anh Nguyen, Y. Pogoryelov, P. K. Muduli, A. Eklund, M. Hoefer, and J. Åkerman, J. Appl. Phys. 115, 172612 (2014).

44. F. Macià, D. Backes, and A.D. Kent, Nature Nanotech. 9, 992 (2014).
45. P.K. Muduli, O.G. Heinonen, and J. Åkerman, J. Appl. Phys. 110, 076102 (2011).

46. J. Sampaio, V. Cros, S. Rohart, A. Thiaville, and A. Fert, Nature Nanotech. 8, 839 (2013).

47. R. Liu, W. Lim, and S. Urazhdin, Phys. Rev. Lett. 114, 137201 (2015).

48. G. Finocchio, V. Puliafito, S. Komineas, L. Torres, O. Ozatay, T. Hauet, and B. Azzerboni, J. Appl. Phys. 114, 163908 (2013).

49. V. Puliafito, G. Siracusano, B. Azzerboni, and, G. Finocchio, IEEE Magn. Lett. 5, 1 (2014).

50. J.C. Slonczewski, J. Magn. Magn. Mater. 195, 261 (1999).

51. Y. Zhou, S. Bonetti, C.L. Zha, and J. Åkerman, New J. Phys. 11, 103028 (2009).

52. Y. Zhou, C.L. Zha, S. Bonetti, J. Persson, and J. Åkerman, Appl. Phys. Lett. 92, 262508 (2008).

53. C. Morrison, J.J. Miles, T.N. Anh Nguyen, Y. Fang, R.K. Dumas, J. Åkerman, and T. Thomson, J. Appl. Phys. 117, 17B526 (2015).

54. T.N. Anh Nguyen, N. Benatmane, V. Fallahi, Y. Fang, S.M. Mohseni, R.K. Dumas, and J. Åkerman, J. Magn. Magn. Mater. 324, 3929 (2012).

55. L. Tryputen, F. Guo, F. Liu, T.N. Anh Nguyen, M.S. Mohseni, S. Chung, Y. Fang, J. Åkerman, Phys. Rev. B 91, 1 (2015).

56. S. Chung, S.M. Mohseni, V. Fallahi, T.N. Anh Nguyen, N. Benatmane, R.K. Dumas, and J. Åkerman, J. Phys. D. Appl. Phys. 46, 125004 (2013).

57. T.N. Anh Nguyen, V. Fallahi, Q. Tuan Le, S. Chung, S.M. Mohseni, R.K. Dumas, C.W. Miller, and J. Åkerman, Magn., IEEE Trans. 50, 2004906 (2014).

58. T.N. Anh Nguyen, R. Knut, V. Fallahi, S. Chung, Q.T. Le, S. Mohseni, O. Karis, S. Peredkov, R. Dumas, C.W. Miller, and J. Åkerman, Phys. Rev. Appl. 2, 044014 (2014).

59. S. Tacchi, T.N. Anh Nguyen, G. Carlotti, G. Gubbiotti, M. Madami, R.K. Dumas, J.W. Lau, J. Åkerman, A. Rettori, and M.G. Pini, Phys. Rev. B 87, 144426 (2013).

60. S. Tacchi, T.N. Anh Nguyen, G. Gubbiotti, M. Madami, G. Carlotti, M.G. Pini, A. Rettori, V. Fallahi, R.K. Dumas, and J. Åkerman, J. Phys. D. Appl. Phys. 47, 495004 (2014).

61. E. Iacocca, R.K. Dumas, L. Bookman, M. Mohseni, S. Chung, M.A. Hoefer, and J. Åkerman, Phys. Rev. Lett. 112, 047201 (2014).

62. M.D. Maiden, L.D. Bookman, and M.A. Hoefer, Phys. Rev. B 89, 180409 (2014). 